\title{
Obsolescencia e idiomática de las referencias bibliográficas de la Revista de la Sociedad Española de Enfermería Nefrológica: años 1998,1999, 2003, 2006 y 2008
}

\author{
Antonio López González - Lorena Díaz Rodríguez - María Veiga Rodríguez - Carmen Ornosa Agra - Oscar \\ Rodríguez Osorio
}

Unidad de Hemodiálisis. U.S.P. Hospital Santa Teresa. A Coruña

\section{Resumen}

Objetivo: llevar a cabo un análisis bibliométrico para conocer y evaluar la obsolescencia e idiomática de las referencias bibliográficas de los artículos publicados en la Revista de la Sociedad Española de Enfermería Nefrológica.

Material y método: se ha realizado un estudio descriptivo longitudinal retrospectivo. Se han utilizado los cuatro números de los volúmenes 1, 2, 6, 9 y 11 de la Revista de la Sociedad Española de Enfermería Nefrológica. Los indicadores bibliométricos analizados han sido la obsolescencia e idiomática.

Resultado: se han revisado 168 artículos, con un total de 1166 referencias bibliográficas, con una mediana de 5, una desviación estándar $\pm 6,54$, un máximo de 39 y un mínimo de 0 referencias bibliográficas por artículo.

El índice total de referencias por artículo ha alcanzado las 6,94 referencias bibliográficas por artículo.

Correspondencia:

Antonio López González

U.S.P. Hospital Santa Teresa (Unidad de Hemodiálisis)

C. / Londres, 2

15009 A Coruña / España

antonlopezglez@hotmail.com
El Índice de Obsolescencia ha sido de 6,1 años, el Índice de Price ha sido del $41,62 \%$ y el Índice de Inmediatez ha alcanzado el 3,4\%. En cuanto al idioma manejado por los autores en el ámbito de la enfermería nefrología, han sido el español (54,87\%; $95 \%$ IC $51,98-57,7)$ y el inglés $(44,13 \% 95 \%$ IC 41,29-47,07) los más utilizados.

Conclusión: el análisis de las referencias bibliográficas de los artículos publicados en la Revista ha presentado parámetros similares a otras revistas sobre ciencias de la salud. Ahora bien, se aprecian unos buenos datos de obsolescencia, situación que revela la buena vigencia de la mayoría de las referencias estudiadas.

PALABRAS CLAVE:

- BIBLIOMETRÍA

- ENFERMERÍA NEFROLÓGICA

- REVISTA

Obsolescence and language use in bibliographical references in the Magazines of the Spanish Nephrology Nursing Society: years 1998,1999, 2003, 2006 and 2008

\section{Abstract}

Aim: to carry out a bibliometric analysis to determine and evaluate the obsolescence and language use 
in the bibliographical references of the articles published in the Magazine of the Spanish Nephrology Nursing Society.

Material and method: a retrospective longitudinal descriptive study was carried out. The four issues of volumes 1, 2, 6, 9 and 11 of the Magazine of the Spanish Nephrology Nursing Society were used. The bibliometric indicators analysed were obsolescence and language.

Result: 168 articles were reviewed, with a total of 1166 bibliographical references, with a median of 5 , a standard deviation of \pm 6.54 , a maximum of 39 and a minimum of 0 bibliographical references per article.

The total index of references per article was 6.94 bibliographical references per article. The Obsolescence Index was 6.1 years, the Price Index was $41.2 \%$ and the Immediacy Index was 3.4\%. As regards the language used by the authors in the sphere of nephrology nursing, Spanish $(54.87 \%$; 95\%CI 51.98-57.7) and English (44.13\% 95\%CI 41.29-47.07) were the most used.

Conclusion: The analysis of the bibliographical references in the articles published in the magazines showed similar parameters to other magazines on health sciences. However, good obsolescence data were obtained, which shows the relevance of most of the references studied.

\section{KEY WORDS:}

- BIBLIOMETRICS

- NEPHROLOGY NURSING

- MAGAZINE

\section{Introducción}

La literatura científica viene experimentando un crecimiento exponencial como ya describió Price en 1956 (citado por López López P)ํㅗ․ Sin embargo, en el ámbito de la enfermería, hasta finales de los años setenta, la producción científica fue muy escasa, y los profesionales se limitaban a colaborar en otras áreas de investigación biomédica. En ellas se limitaban a tareas mecánicas, habitualmente relacionadas con la recogida y tabulación de los datos. Esta situación ha ido cambiando gradualmente a lo largo de los últimos 30 años con la incorporación de los estudios de enfermería en la Universidad (1977) y con la aplicación de políticas de calidad por parte del sistema sanitario, que obligan a hacer un seguimiento y evaluación de las actividades de enfermería ${ }^{2}$. Actualmente este incremento de la actividad científica enfermera se ha podido ver favorecida por el uso de las taxonomías en el trabajo diario, la incorporación de la enfermería basada en la evidencia, la cual aporta una reducción en la variabilidad en la práctica clínica ya que las decisiones serán tomadas teniendo en cuenta el mejor conocimiento disponible, y la investigación cualitativa, que nos proporciona nuevas dimensiones sobre la conceptualización de problemas de enfermería?

Este apogeo de la actividad investigadora por parte de la enfermería da pie a la aparición de las primeras publicaciones. Estas se han convertido en elementos imprescindibles a la hora de establecer estrategias de comunicación de la información científica, ya que a través de ellas pueden transmitirse los conocimientos obtenidos ${ }^{4}$ (en ciencias de la salud el $80 \%$ de las investigaciones se transmiten a través de publicaciones ${ }^{5}$ ).

En el ámbito de la enfermería nefrológica en España, destaca la gran labor de La Revista de la Sociedad Española de Enfermería Nefrológica. Esta revista, en su actual formato, se publica desde 1998, pero representa la continuidad de otra denominada BISEAN, que fue el primer órgano de difusión de la Sociedad Española de Enfermería Nefrológica, fundada en 1975 con el objetivo de promover el progreso científico y el intercambio de experiencias, con vistas a lograr el mejor cuidado de los enfermos renales.

Tanto el crecimiento de la producción científica como el desarrollo de revistas $y$, actualmente, el auge de las bases de datos bibliográficas automatizadas han potenciado el uso, por parte de las ciencias de la salud, de la bibliometría y la generación de indicadores para medir los resultados de la actividad científica y tecnológica. Estos estudios se basan en el análisis estadístico de datos cuantitativos procedentes de la literatura científica, constituyendo en la actualidad 
la herramienta esencial para el conocimiento de la actividad investigadora, aportando datos sobre la situación científica de un país o tema de investigación, y permitiendo evaluar el rendimiento de la actividad científica y su impacto en la comunidad. El primer estudio bibliométrico fue presentado en 1917 por Coles y Eales en Science Progress, en él analizaban publicaciones sobre anatomía comparativa entre 1543 a 1860. La primera tesis doctoral sobre este campo aparece en $1970^{6}$.

El objetivo del presente trabajo es analizar y evaluar la obsolescencia e idiomática de las referencias bibliográficas de los artículos publicados en La Revista de la Sociedad Española de Enfermería Nefrológica (SEDEN)

\section{Material y métodos}

Tipo de estudio: estudio descriptivo longitudinal retrospectivo

Muestra a estudio: las referencias bibliográficas de los artículos publicados en la Revista de la SEDEN en los años 1998, 1999, 2003, 2006 y 2008.

Justificación de la muestra: hemos elegidos el año 1998 ya que corresponde al primer año en el que se publicó la Revista de la SEDEN en su actual formato y el 2008 por ser el último año disponible en la web de la Revista de la SEDEN (www.revistaseden.org) en el momento de realización del estudio (marzo-abril de 2008). El resto de los años (1999, 2003 y 2006) han sido elegidos al azar.

Criterios de inclusión: estar publicados en los años 1998,1999, 2003, 2006 y 2008.

Criterios de exclusión: en relación a los artículos, por un lado, se han excluido las editoriales ya que este tipo de trabajos, al expresar opiniones e interpretaciones, no suelen ir acompañados de bibliografía. Por otro lado, se han excluidos los índices bibliográficos ya que estos últimos nos introducirían un sesgo en función de la realidad de esa búsqueda; además, de ellos no se extrae ningún texto.

En cuanto a las referencias bibliográficas, se han excluido aquellas en las que no estaba reflejada la fecha.
Mediciones/intervenciones: una vez realizada la búsqueda bibliográfica previa, se ha registrado información, por medio de la revisión manual de todos los artículos incluidos en el estudio, de los siguientes grupos de variables:

1. Relacionadas con la revista: identificación de la revista, año, volumen y número de la publicación.

2. Relacionada con los artículos: número de artículos

3. Relacionadas con la bibliografía: número de referencias, año de publicación e idioma de publicación.

Como instrumentos de medición hemos utilizado los siguientes indicadores bibliométricos:

- Índice de referencias por artículo, que forma parte de un conjunto de indicadores denominados de producción, los cuales miden cuantitativamente la información científica producida.

- Índices de consumo, que son utilizados para cuantificar el consumo de la producción científica. Hemos elegido el Índice de Prince (porcentaje de referencias con antigüedad menor de cinco años), el Índice de Inmediatez (porcentaje de referencias referidas con antigüedad menor a un año) y el Índice de Obsolescencia o vida media o semiperiodo de Burton y Kleber (la edad del 50\% de las citas publicadas en una revista en el apartado de referencias bibliográficas).

Análisis estadístico: con la ayuda del paquete estadístico SPSS, se ha hecho un análisis descriptivo de los datos. Las variables cualitativas se presentan con cifras absolutas y porcentajes con su correspondiente intervalo de confianza al 95\%. Las variables cuantitativas se presentan como una media, mediana, desviación estándar y máximo-mínimo.

\section{Resultados}

En los años 1998, 1999, 2003, 2006 y 2008 se han publicado en La Revista de la Sociedad Española de Nefrología 193 artículos que contenían 1221 referencias bibliográficas. De ellos hemos analizado 168, con un total de 1166 referencias bibliográficas, con 
una mediana de 5, una desviación estándar $\pm 6,54$, un máximo de 39 y un mínimo de 0 referencias bibliográficas por artículo.

En cuanto a la producción (tabla 1), el Índice total de referencias por artículo indica que, en promedio, los artículos publicados en la Revista de la SEDEN contenían alrededor de siete referencias bibliográficas. Este valor se ha mantenido prácticamente constante en todos los volúmenes analizados con la excepción del Volumen 1.

\begin{tabular}{|c|c|c|c|}
\hline $\begin{array}{l}\text { Volumen y } \\
\text { Números }\end{array}$ & $\begin{array}{l}\text { Número de } \\
\text { artículos }\end{array}$ & Referencias & $\begin{array}{l}\text { Índice de } \\
\text { referencias } \\
(95 \% \text { IC) }\end{array}$ \\
\hline $\begin{array}{l}11(2008) \\
N^{\circ} 1,2,3,4\end{array}$ & 33 & 244 & $\begin{array}{c}7,39 \\
(5,74-9,65)\end{array}$ \\
\hline $\begin{array}{c}9(2006) \\
N^{\circ} 1,2,3,4\end{array}$ & 33 & 240 & $\begin{array}{c}7,27 \\
(5,30-9,23)\end{array}$ \\
\hline $\begin{array}{l}6(2003) \\
N^{\circ} 1,2,3,4\end{array}$ & 30 & 211 & $\begin{array}{c}7,03 \\
(4,26-9,79)\end{array}$ \\
\hline $\begin{array}{l}2(1999) \\
N^{\circ} 1,2,3,4\end{array}$ & 39 & 282 & $\begin{array}{c}7,23 \\
(4,61-9,71)\end{array}$ \\
\hline $\begin{array}{l}1(1998) \\
N^{0} 1,2,3,4\end{array}$ & 33 & 189 & $\begin{array}{c}5,72 \\
(3,34-8,10)\end{array}$ \\
\hline TOTAL & 168 & 1162 & $\begin{array}{c}6,94 \\
(6,56-7,32)\end{array}$ \\
\hline
\end{tabular}

Tabla 1. Distribución de artículos, referencias bibliográficas e índice de referencias por artículo

Por otra parte, relativo al consumo de información, hemos obtenidos los siguientes resultados (tabla 2). El Índice de Price para la totalidad de las referencias bibliográficas ha alcanzado el $41,62 \%$. El valor de este índice ha oscilado desde un máxim o de 55,13\% en el Volumen 2 hasta un mínimo de 34,92\% en el Volumen 1 (figura 1). El Índice de Obsolescencia 0 vida media ha sido de 6,1 años. Se ha evidenciado una tendencia al descenso de la vida media con el paso de los años. Por último, el Índice de Inmediatez para el total de referencias ha alcanzado el 3,4\%, siendo en el Volumen 2 donde la inmediatez ha sido más alta y en el Volumen 11 donde ha sido más baja.

\begin{tabular}{|c|c|c|c|}
\hline Año & $\begin{array}{l}\text { Vida media } \\
\text { (años) }\end{array}$ & $\begin{array}{l}\text { Índice de } \\
\text { Price (\%) }\end{array}$ & $\begin{array}{c}\text { Índice de } \\
\text { Inmediatez (\%) }\end{array}$ \\
\hline $\begin{array}{l}2008 \text { (Vol } \\
11) \\
95 \% \text { IC }\end{array}$ & $\begin{array}{c}5,93 \\
(4,83-4,70)\end{array}$ & $\begin{array}{c}44,17 \\
(37,7-50,7)\end{array}$ & $\begin{array}{c}2,5 \\
(0,9-5,3)\end{array}$ \\
\hline $\begin{array}{c}2006 \text { ( Vol 9) } \\
95 \% \text { IC }\end{array}$ & $\begin{array}{c}6,13 \\
(4,56-7-70)\end{array}$ & $\begin{array}{c}38,33 \\
(32,15-44,8)\end{array}$ & $\begin{array}{c}3,75 \\
(1,7-6,9)\end{array}$ \\
\hline $\begin{array}{c}2003 \text { ( Vol 6) } \\
95 \% \text { IC }\end{array}$ & $\begin{array}{c}6,40 \\
(5,47-7,33)\end{array}$ & $\begin{array}{c}35,55 \\
(29,09-42,4)\end{array}$ & $\begin{array}{c}3,32 \\
(1,3-6,7)\end{array}$ \\
\hline $\begin{array}{c}1999(\mathrm{Vol} 2) \\
95 \% \mathrm{IC}\end{array}$ & $\begin{array}{c}4,74 \\
(3,20-6,26)\end{array}$ & $\begin{array}{c}55,13 \\
(46,1-58,8)\end{array}$ & $\begin{array}{c}4,26 \\
(2,21-7,3)\end{array}$ \\
\hline $\begin{array}{c}1998(\text { Vol } 1) \\
95 \% \text { IC }\end{array}$ & $\begin{array}{c}7,30 \\
(5,59-8,29)\end{array}$ & $\begin{array}{c}34,92 \\
(28,14-42,27)\end{array}$ & $\begin{array}{c}3,17 \\
(1,16-6,78)\end{array}$ \\
\hline $\begin{array}{c}\text { TOTAL } \\
\text { (95\% IC) }\end{array}$ & $\begin{array}{c}6,1 \\
(5,72-6,48)\end{array}$ & $\begin{array}{c}41,62 \\
(41,01-47,5)\end{array}$ & $\begin{array}{c}3,4 \\
(2,4-4,64)\end{array}$ \\
\hline
\end{tabular}

Tabla 2. Comparativa anual de las referencias bibliográficas

En cuanto a los idiomas expresados en las referencias bibliográficas de los artículos analizados se ha evidenciado el predominio del español presente en un $54,87 \%(95 \%$ IC $51,98-57,7)$ seguido del inglés presente en un $44,13 \%$ (95\% IC 41,29-47,07). El francés $(0,40 \% ; 95 \%$ IC $0,13-0,99)$ y el catalán $(0,6 \% ; 95 \%$ IC $0,18-1,11)$ han sido los otros idio-

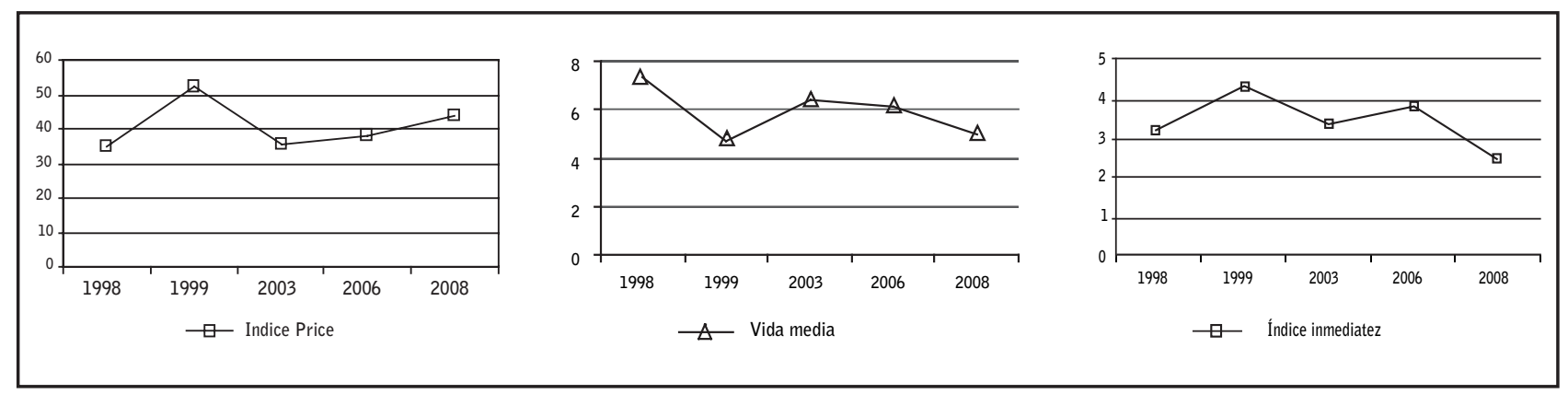

Figura 1. Comparativa anual de las referencias bibliográficas 
mas presentes en el análisis pero en menos medida (tabla 3).

\begin{tabular}{|c|c|c|c|c|c|}
\hline $\begin{array}{c}\text { Volumen y } \\
\text { Números }\end{array}$ & Español & Ingles & Francés & $\begin{array}{c}\text { Otros } \\
\text { (Catalán) }\end{array}$ & Total \\
\hline $\begin{array}{c}11(2008) \\
N^{0} 1,2,3,4\end{array}$ & 147 & 97 & 0 & 0 & 244 \\
\hline $\begin{array}{c}9(2006) \\
N^{0} 1,2,3,4\end{array}$ & 153 & 84 & 0 & 3 & 240 \\
\hline $\begin{array}{c}6(2003) \\
N^{0} 1,2,3,4\end{array}$ & 111 & 100 & 0 & 0 & 211 \\
\hline $\begin{array}{c}2(1999) \\
N^{0} 1,2,3,4\end{array}$ & 139 & 139 & 2 & 2 & 282 \\
\hline $\begin{array}{c}1(1998) \\
N^{0} 1,2,3,4\end{array}$ & 90 & 95 & 3 & 1 & 189 \\
\hline \begin{tabular}{c} 
TOTAL \\
\hline
\end{tabular} & 640 & 515 & 5 & 6 & 1166 \\
\hline
\end{tabular}

Tabla 3. Distribución de referencias bibliográficas según el idioma

\section{Discusión}

La evaluación de una revista, desde la perspectiva cuantitativa, es tarea complicada, en la cual los análisis bibliométricos son uno de los instrumentos más útiles. Estos llevan asociados la utilización y aplicación tanto de indicadores como de modelos matemáticos cuyo objetivo es obtener datos que permitan aportar conocimiento en relación a la evolución de una determinada producción científica y conocer igualmente su calidad.

En relación al consumo de producción científica, la media de 6,94 referencias bibliográficas por artículo que ofrece el total de los artículos analizados es inferior al patrón habitual de las revistas científicas, que en el año 1994 se situaba en 157 , sin embargo, esta cerca del resultado que presenta el mapa bibliométrico elaborado por Camí para el conjunto de España en el periodo de 1994-2002, que se sitúa en 7,6 referencias por documento ${ }^{8}$.

Por otro lado, un problema inseparable del crecimiento científico es el de la obsolescencia o envejecimiento de la literatura científica. En nuestro estudio se puede observar como la obsolescencia de las referencias bibliográficas ha sufrido un ligero descenso con el paso de los años. Además, hay que tener en cuenta que nuestra especialidad presenta lazos de unión con la fisiología cuya obsolescencia es alta (como es común en las ciencias básicas) por lo que es lógico que nuestra obsolescencia se vea por este motivo ligeramente aumentada.

Se han descubierto artículos que utilizaban un alto porcentaje de referencias con más de 15 años de antigüedad, con lo que se podría concluir que estos artículos están desfasados en el momento de su publicación. Sin embargo, el hecho que la obsolescencia en La Revista de la Sociedad Española de Enfermería Nefrológica sea de 6,1 años no implica que en futuros estudios solo se pueda utilizar referencias bibliográficas que tengan esa antigüedad. Este índice lo que demuestra es la tendencia a envejecer de la literatura manejada por los profesionales y por tanto su pérdida de utilidad.

En definitiva, el análisis de la obsolescencia nos ofrece datos similares o algo mejores en relación a otros trabajos ${ }^{8-10}$, lo que indica la buena actualidad de las referencias bibliográficas utilizadas en este campo.

Por último, el análisis idiomático nos permite conocer la capacidad que tiene el autor de comprender y trabajar en distintas lenguas. Este estudio nos muestra que la lengua española es la más utilizada seguida de la inglesa. El predominio del castellano se podría explicar en que la Revista de la SEDEN se edita en España, donde la lengua oficial es el castellano, y por tanto, es posible que los investigadores prefieran seguir con más interés las investigaciones vinculadas a este idioma. Además, al ser la mayoría de los investigadores españoles, se supone que se sienten más culturalmente familiarizados con el idioma en el que fueron educados. Por otra parte, el elevado uso del inglés se pude deber a la gran cantidad de información que se publica en este idioma, a que las fuentes de información con más visibilidad y de mayor impacto se publican en ingles; y por último, y cada vez más común, a la tendencia a citar trabajos en ingles (a pesar de no haberlos consultado) con el fin de aportarle al texto un carácter más "impactante".

\section{Bibliografía}

1. López López P. Bibliometría: la medida de la información. En: López Yepes J (coord.). Manual de información y Documentación. Madrid: Pirámide, 1996. p. 488-411. 
2. Morales Asencio JM. ¿Qué aporta el concepto de evidencia científica a la práctica clínica de los cuidados? Index de Enfermería (edición digital) 2003 [Consultado el 23 de abril 2009]; 40-41. Disponible en http://www.index-f.com/index-enfermeria/40-41revista/40-41_articulo_35-40.php.

3. Tirado Pedregosa, G. Actividad Científica en Enfermería: ¿para cuándo su reconocimiento? Enfermería Comunitaria (rev. digital) 2007,3(2). Disponible en http://www.index-f.com/comunitaria/v3n2/ec2597. php [Consultado el 23 de abril 2009].

4. Jiménez Hernández J A, Ayuso García $M^{a} D, M u-$ rillo Murillo $R$, Guillén Ríos Juan $F$. Evolución de las publicaciones periódicas españolas de enfermería: A quantitative analysis. Index de Enfermería (edición digital) 2007 [Consultado el 23 de abril 2009]; 16(56): 73-78. Disponible en: http://scielo. isciii.es/scielo.php?script=sci_arttext\&pid=S1132$12962007000100018 \& \operatorname{lng}=e s \& n r m=i s o$.

5. Chain Navarro C M, Lorente Gallego $A M^{a}$, Flores JA. Análisis bibliométrico de la revista Rol de Enfermería (2000-2004). Rev ROL Enferm 2007; 30:18-22.
6. Zulueta MA, Cabrera A, Bordons M. Identificación y estudio de grupos de investigación a través de indicadores bibliométricos. Rev Esp Doc Cient 1999; 22:333-47.

7. López Piñeiro JM, Terrada ML. El consumo de información nacional y extranjera en las revistas medicas españolas: un nuevo repertorio destinado a su estudio. Med Clin (Barc). 1994; 102: 104-12.

8. Camí J, Méndez Vázquez R, Suñén Piñol E. Mapa bibliométrico de España 1994-2002: biomedicina y ciencias de la salud. Med Clin (Barc) 2005; 124(3):93-101.

9. Villar Álvarez F, Estrada Lorenzo JM, Pérez Andrés C, Rebollo Rodríguez MJ. Estudio bibliométrico de los artículos originales de la revista española de salud pública (1991-2000). Parte tercera: análisis de las referencias biblográficas. Rev Esp Salud Pública 2007; 81(3):247-59.

10. Fernández Baena MJ. Las referencias bibliográficas de los artículos publicados en la Revista Española de Anestesiología y Reanimación. Estudio del periodo 19992003. Rev Esp Anestesiol Reanim 2006; 53(5):283-8. 